\title{
Tangible Images: Bridging the Real and Virtual Worlds
}

\author{
James A. Ferwerda and Benjamin A. Darling \\ Munsell Color Science Laboratory, Chester F. Carlson Center for Imaging Science, \\ Rochester Institute of Technology \\ \{jaf, bad4235\}@cis.rit.edu
}

\begin{abstract}
In this paper we describe our efforts to create tangible imaging systems that provide rich virtual representations of real-world surfaces. Tangible imaging systems have three main properties: 1) the images produced must be visually realistic; 2) the images must be responsive to user interaction; and 3) the images must be situated, appearing to be integrated with their environments. Our current system, based on a computer, LCD display, light and position sensors, and graphics rendering tools meets all these requirements; supporting the accurate simulation of the appearances of surfaces with complex textures and material properties, and allowing users to interact with and experience these virtual surfaces as if they were real ones. We first describe the components of our current system and its implementation. We then illustrate the system's capabilities for simulating the appearances and behaviors of real-world surfaces. Finally we describe some potential applications of tangible imaging systems and discuss limitations and future work.
\end{abstract}

Keywords: tangible interfaces, realistic image synthesis, surface appearance.

\section{Introduction}

Surfaces cover everything, and it is through the interaction of light with these surfaces and our eyes that we are able to visually perceive the properties of the world. We create images through graphics or photography to record the properties of these surfaces and to communicate them to others, and images can serve quite well as visual representations, but still the visual information provided by an image of a surface is not the same as that provided by the surface itself.

We get a lot of information from interacting with surfaces, either through direct manipulation, or through observing a surface from different viewpoints, and conventional images do not support either of these behaviors. For this reason we have been working to create tangible imaging systems [1-4], that harness the power of digital modeling, computer graphics, and modern mobile computing platforms to produce new kinds of images that look and behave much more like the surfaces they represent.

Figure 1 shows one implementation of a tangible imaging system, the tangiBook, based on an off-the-shelf laptop computer. Computer graphics hardware and custom software in the device allows a 3D model of an oil painting to be rendered to the screen in real-time, with realistic lighting. Orientation sensors and observer tracking 
systems change the rendering as the device or observer move. The experience is similar to that of holding and observing a real painting.

In this paper we describe our efforts to create tangible imaging systems like the one described above that serve as rich digital representations of real surfaces. We believe that to achieve this goal the systems must have three properties. First, the images produced by the systems must be realistic, accurately representing the shapes and material properties of the modeled surfaces. Second the images must be responsive, changing appearance appropriately with direct manipulation and changes in observer viewpoint. And finally, the images must be situated, appearing to be an integral part of the observer's environment.

In the following sections we first discuss prior efforts in this area by others and ourselves. We then describe the components of our current system that meets all of the above-mentioned requirements, and we illustrate its capabilities. Finally, we outline potential applications of tangible imaging systems and discuss ongoing and future work.

\section{Prior Work}

There has been strong interest in developing natural interfaces for interacting with virtual environments since the earliest days of computer graphics. Sutherland and colleagues, along with laying the foundations for 3D graphics rendering, did pioneering work on developing head-mounted display systems and 3D input devices [5,6] Significant advances have been made in both areas since that time [7-12].

An alternative approach that supports direct interaction with virtual environments is the CAVE system [13]. In this system, users are surrounded by projection screens that display computer graphics renderings. Stereoscopic eyewear, user position tracking, and gestural interfaces have all been added to different CAVE systems to increase feelings of immersion and support for direct interaction. Another projectorbased approach is represented by the shaderLamps and iLamps systems developed by Raskar et al. [14-17], as well as similar work by Bimber and colleagues. In these systems, warped CG images are projected onto real three-dimensional objects to give the observer the experience of real objects with surface properties that can be changed under computer control.

A third major approach for supporting direct interaction with virtual environments incorporates spatially aware displays and tangible interfaces. In the Chameleon systems $[18,19]$ computer monitors were fitted with 6 degree-of-freedom trackers to create spatially-situated displays whose virtual content changed depending on their real-world positions. The Virtual Mirror system [20] took the concept one step further by incorporating a video camera pointed toward the user to create the impression of a real mirror. This system also allowed interactive viewing of reflective daguerreotype images [21]. A significant feature of all these systems is their support for direct manipulation through tangible interfaces. The strength of tangible interfaces, is that the affordances of the systems (lifting, tilting, and rotating the display) support rich and natural modes of interaction with the virtual content $[22,23]$. 

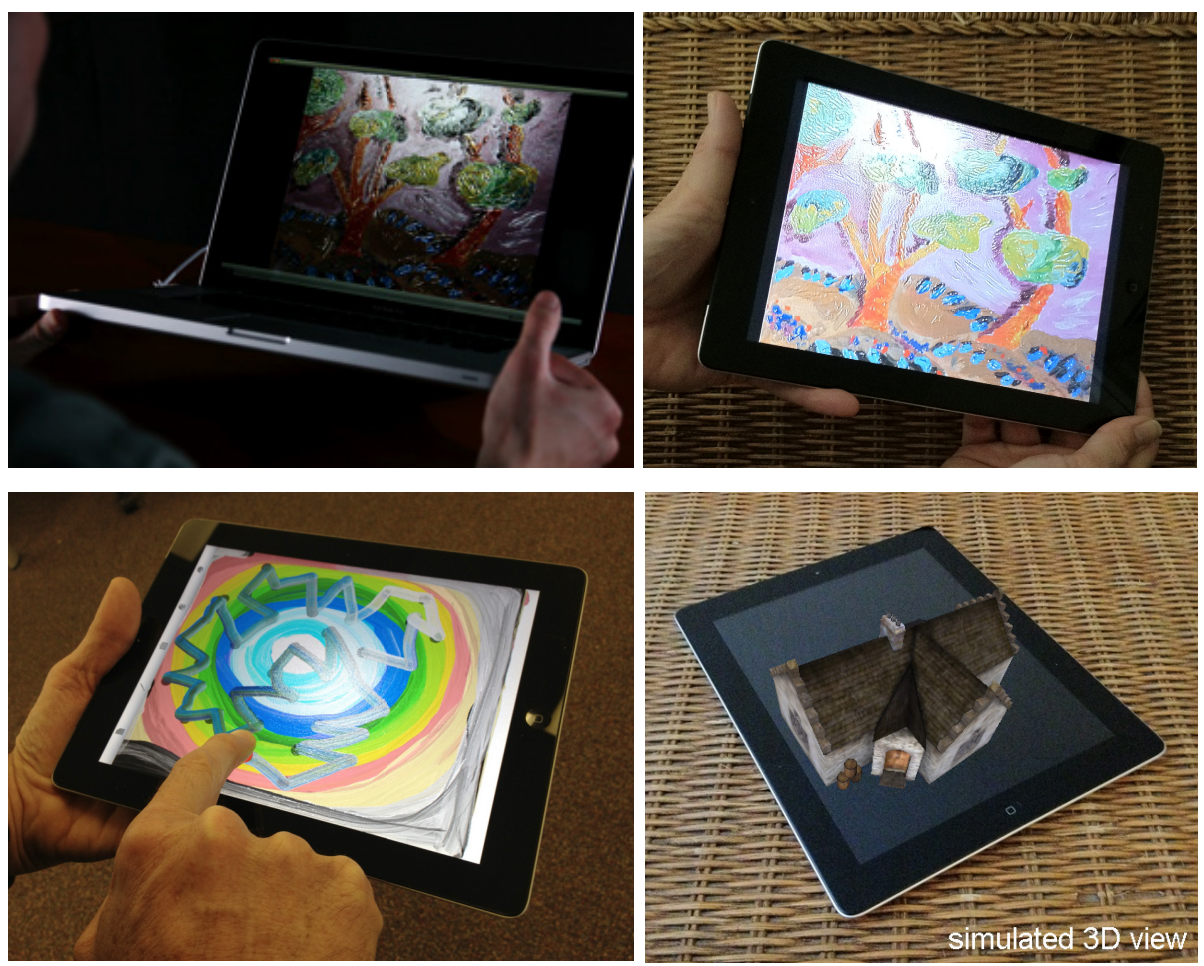

Fig. 1. Tangible imaging systems (left to right, top to bottom): The first generation tang iBook system developed using an off-the-shelf laptop computer; the tangiView system implemented on a tablet device; the tangiPaint application that allows direct creation of and interaction with rich digital paintings; the phantoView system that allows tangible interaction with threedimensional object models

\subsection{Our Prior Work}

As evidenced by the work described above, creating a virtual environment system that can simulate the experience of interacting with real things requires real-time realistic rendering and support for natural modes of user interaction. Our goal in designing the tangiBook [1] was to create a proof-of-concept tangible imaging system that achieved these goals for graspable surfaces such as paintings, prints, etc.. The tangiBook was based on an off-the-shelf laptop computer (Apple MacBook Pro) that incorporated all the necessary components. The laptop's high-resolution LCD display screen backed by graphics rendering hardware and software supported realistic rendering, and its built-in accelerometer and camera supported direct manipulation and user tracking. As shown in Figure 1 (top, left), tilting the laptop or moving in front of it produced realistic changes in the appearance (color, gloss, texture) of the rendered surface. While the capabilities of the tangiBook were promising, the laptop form-factor was a bit awkward to use, so when tablet computers became available we implemented a second-generation system called tangiView [2]. Figure 1 (top, right) shows how the 
tablet device provides a more natural interface for the low relief surfaces we were interested in simulating. Figure 1 (bottom, left) shows the tangiPaint [3] system, developed on the same tablet platform that allows users to create their own "paintings" and view them using the tangible imaging platform. Sophisticated canvas, paint, and brush models produce paintings with realistic color mixing, gloss, and brushstroke texture. Finally, we have recently taken the tangible imaging concept into 3D with the development of the phantoView [4] application shown in Figure 1 (bottom, right). Rendering using anamorphic stereo projections and red/blue anaglyphs provides and the appearance of objects that sit on the tablet screen. Device tracking allows the user to view different sides of the object by rotating the tablet around its vertical axis.

While the systems described above illustrate the capabilities and potential of tangible imaging systems, all the images produced by these systems are just pretty pictures. To be useful in domains such as appearance design and communication, electronic commerce, or digital archiving, it is not enough that the images look good or produce compelling experiences, they must be measurably accurate representations of real surfaces. In the following sections we describe our efforts to develop a system that can meet these goals.

\section{System Design}

The goal in creating tangible imaging systems is to develop imaging technology that bridges the real and virtual worlds. To reach this goal we identified three requirements: 1) the images produced by the systems must be realistic, accurately representing the shapes and material properties of the modeled surfaces; 2) the images must be responsive, changing appearance appropriately with direct manipulation and changes in observer viewpoint; and 3) the images must be situated, appearing to be an integral part of the observer's environment. In the following sections we describe how we have designed a system that meets each of these requirements.

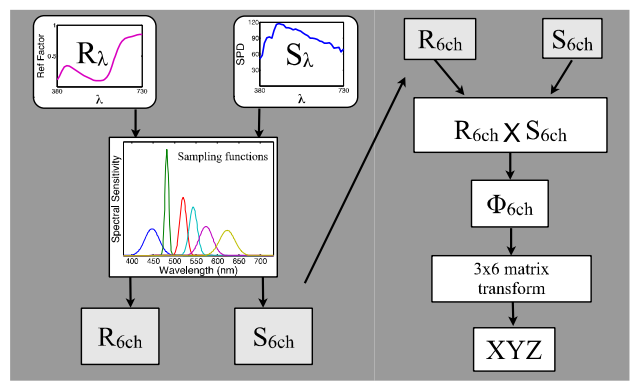

Fig. 2. Six channel multispectral rendering pipeline implemented in the system. Described in detail in [24].

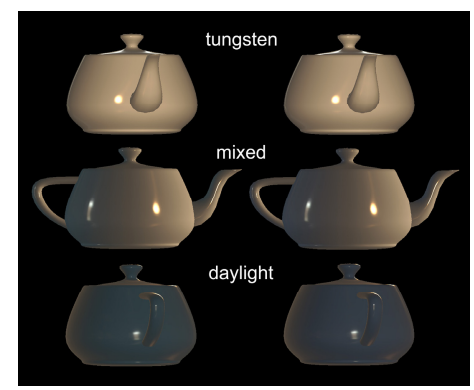

Fig. 3. Metameric matches and failures under different illuminants as rendered by the six-channel pipeline 


\subsection{Realism}

To create displayed images that accurately represent the shapes and material properties of real surfaces we need to first model these properties, and then render these models using advanced computer graphics methods. Our surface models include information about surface color, gloss and texture.

Representing Color: In the extreme, accurately modeling and rendering surface color requires representing the reflectance spectrum of the surface, the emission spectrum of the light source illuminating the surface, and the response properties of the display system. For the sake of performance, these full spectral calculations are typically reduced to products of normalized RGB values used to represent surface and source properties that are then scaled and sent as RGB digital counts to the display. While this approach is common, it can lead to gross errors in color rendering.

In our system, to preserve color accuracy while also supporting real-time graphics performance, we have developed an abridged spectral rendering pipeline [24]. Figure 2 shows the pipeline, where surface and source spectra are each coded into signals in six channels, which can then used for colorimetrically-accurate real-time rendering performed by commodity graphics hardware (GPUs). Output of the system is illustrated in Figure 3, where the metameric colors of the teapots are correctly rendered under a variety of illumination conditions.

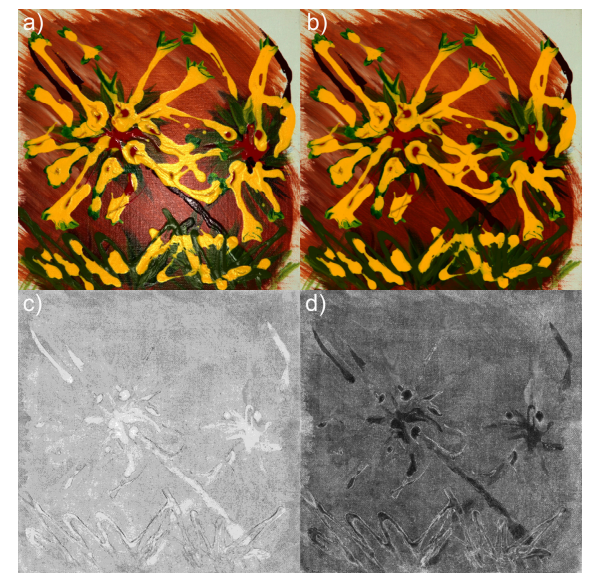

Fig. 4. System representation of material properties a) full rendering, b) diffuse color, c) specular lobe magnitude, d) specular lobe spread

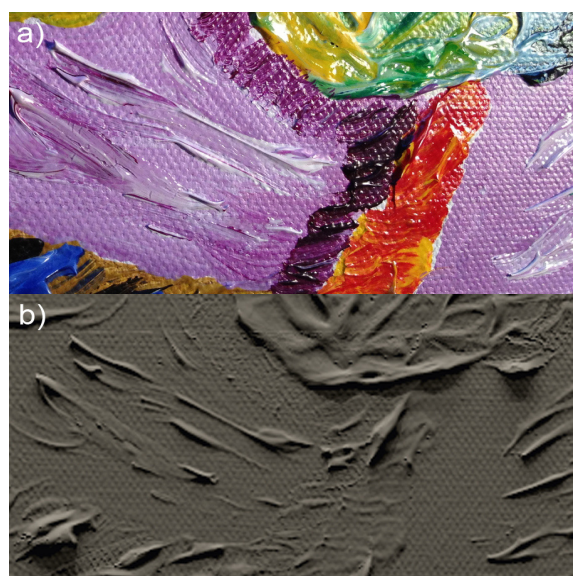

Fig. 5. System representation of texture. a) full rendering, b) texture-only rendering showing shading and shadowing effects

Representing Gloss: Real surfaces vary in gloss as well as color. Gloss is related to the directional reflectance properties of surfaces that can be measured with gonioreflectometers or similar instruments and represented by bi-directional reflectance distribution functions (BRDFs). BRDFs are often characterized in terms of their diffuse (uniform, Lambertian) and specular (directional) components. In our 
system we model the BRDFs of measured surfaces using the Ward [25] light reflection model that has three parameters, $\rho_{d}-$ a factor representing the uniform diffuse component, $\rho_{\mathrm{s}}-$ a factor representing the magnitude of the specular component, and $\alpha-$ a factor representing the spread of the specular lobe. To model complex surfaces with spatially-varying reflectance properties, we store the Ward parameters in three image maps. These parameters are then used to render realistic images of the surfaces. Figure 4 shows painting with complex spatially-varying color and gloss properties, and the three parameter maps used to represent these properties.

Representing Texture: In addition to color and gloss, real surfaces also have textures: complex variations in 3D surface height and orientation that interact with the other surface properties and surface illumination to produce the patterns of shading and shadowing that give real surfaces their rich tactile qualities. Surface texture can be measured using a variety of mechanical or optical metrology techniques that produce maps of surface orientation and height. In our system we use surface normal maps to represent surface orientation and height maps to represent surface height at each surface point. The surface normal maps are used during rendering to calculate surface shading, and the effects of changing surface orientation with respect to light sources. The height maps are used to calculate horizon maps that are used during rendering to estimate light source occlusion to produce shadowing effects. Figure 5 shows a painting with complex textural properties related to the canvas and brushstrokes and a rendering produced by our system of the shading and shadowing effects produced by this texture.
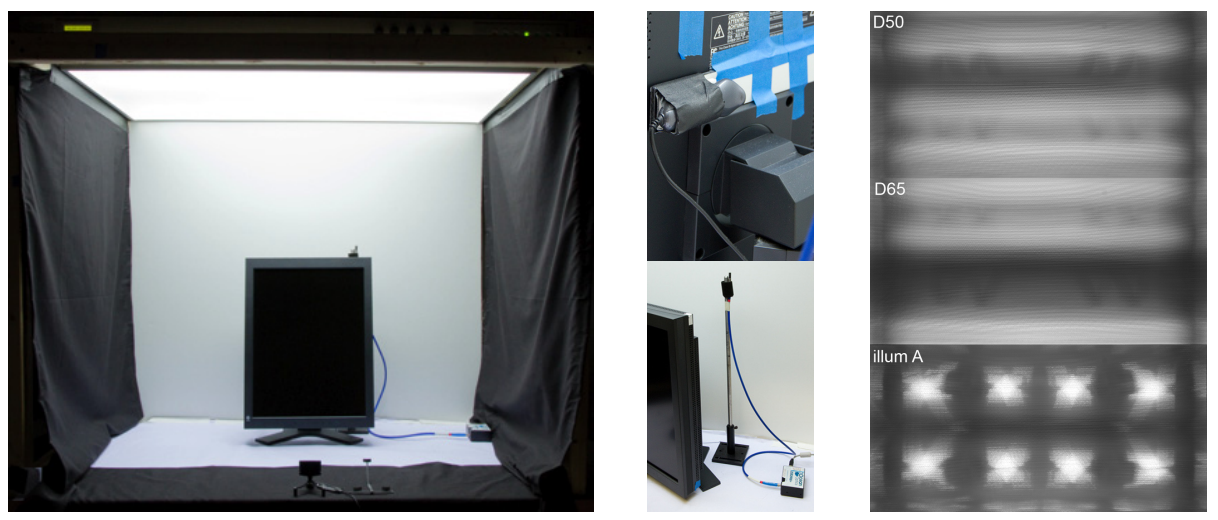

Fig. 6. System hardware (clockwise from left) LCD monitor in light booth with IR tracker, orientation tracker, light booth luminaires, digital spectrometer

\subsection{Responsiveness}

The second requirement we set for our system is that as with real surfaces, the images should be responsive to both direct manipulation and changes in observer viewpoint. To achieve this goal we started with the hardware platform illustrated in Figure 6. For the display we chose an Eizo RX220 medical LCD monitor for its luminance range, 
color gamut, uniformity, and resolution. The monitor was mounted on a stand that allowed it to be easily grasped and tilted and rotated by a user. The monitor and stand were placed in a light booth with selectable luminaires and achromatic walls and floor.

To track the orientation of the monitor we attached an Action XL 3DOF motion sensor to the case. To track the position of the user with respect to the monitor we used a Natural Point IR tracker. The information provided by these sensors was fed to our rendering engine and used to calculate the correct surface appearance given the pose of the monitor screen in the light booth and the viewpoint of the user. The sensing and rendering system operates at interactive rates to allow the user to actively manipulate the surface/image and dynamically change their viewpoint.

\subsection{Situatedness}

The third requirement we set for our system is that as with real reflective surfaces, the images should be situated, and appear to be an integral part of the environment. To meet this requirement the image needs to appear to be illuminated by the light sources in the environment and to change appropriately with changes in the illumination. To achieve this goal we used an Ocean Optics USB2000+ spectrometer to provide realtime sensing of the spectra of the light booth illuminants. Figure 6 shows the device positioned behind the monitor in the light booth. Spectral measurements provided by the device were fed to the multi-spectral color rendering system described earlier to provide colorimetrically accurate renderings of the modeled surfaces under different illuminants.

To provide the experience of a situated surface, the spatial properties of illumination and surface interaction must also be modeled and rendered. To achieve this goal we used HDR imaging techniques to characterize the spatial distributions of the light booth luminaires. Representative image are shown in Figure 6. Information about the illumination distributions was fed into the rendering engine and used to render images whose reflectance patterns are radio metrically accurate with respect to the real sources.

\section{System Capabilities}

The system described above meets the three requirements we set out for tangible imaging systems. First, the images produced by the system are realistic, and faithfully represent the shapes and material properties of modeled surfaces. Second, the images are responsive, and change appearance appropriately with direct manipulation and changes in user viewpoint. And third, the images are situated with respect to the scene illumination and the observer and appear to be an integral part of the user's environment. In the following sections we describe and illustrate the capabilities of the system for simulating reflective surfaces. 

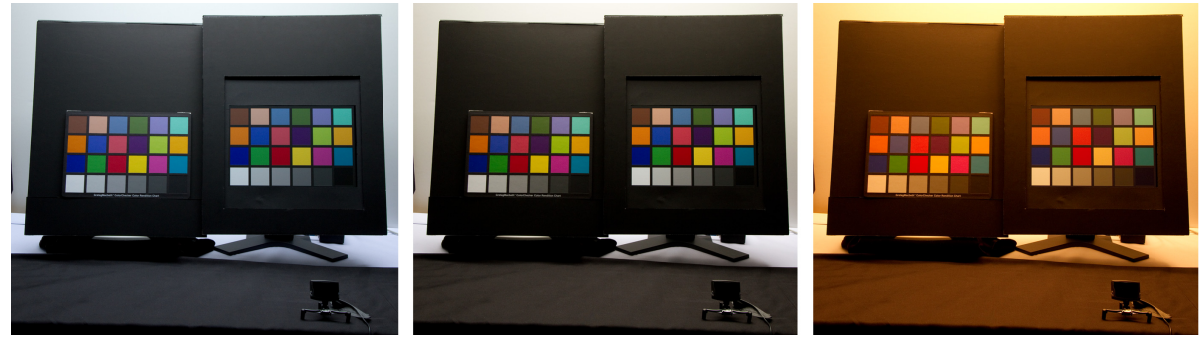

Fig. 7. Simulating color. In each panel the real Macbeth Color Checker Chart is on the left, the image produced by the system is on the right. Note that the simulated colors change appropriately with respect to the different illuminants.
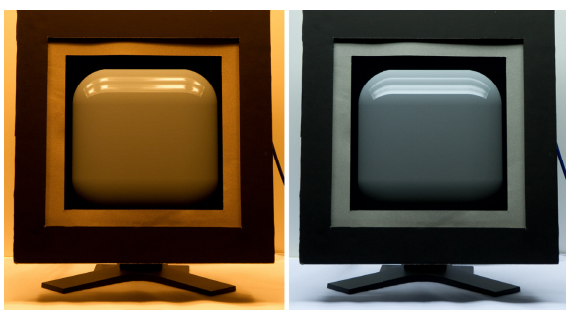

Fig. 8. Simulating gloss. Note that the colors and patterns of the glossy highlights are correct with respect to the different light booth luminaires

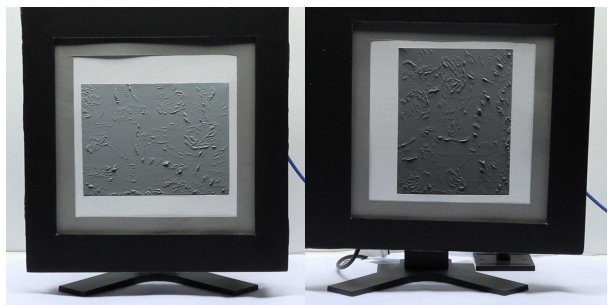

Fig. 9. Simulating texture. Note the surface shading and shadowing effects shown in the two panels and that the effects are correct with respect to the surface's orientation to the light source

Color: Figure 7 shows the color simulation capabilities of the system. Each subimage shows a real Macbeth Color Checker chart on the left and the chart image produced by the system on the right. Note first that the color rendering is quite accurate in part due to the 6-channel color workflow used in the system. Note also that because the system can sense the spectrum of the illuminant, the colors in the simulated chart change appropriately for the different illuminants available in the light booth (the actual appearance differences of the charts when directly viewed are not as extreme as shown here due to chromatic adaptation effects).

Gloss: Figure 8 shows the ability of the system to simulate the appearance of glossy surfaces. The reflectance properties of the surfaces were defined using the Ward light reflection model that was then used in conjunction with the spectrally and spatially characterized model of the booth luminaires to produce the renderings shown. Note that the hues of the neutral surfaces change appropriately for the different illuminants, and that the reflection patterns in the surfaces are correct for the patterns of lights in the different luminaires. Although it cannot be shown in still images, because of the system's IR tracker, the locations of the surface highlights also change appropriately with observer movement. 


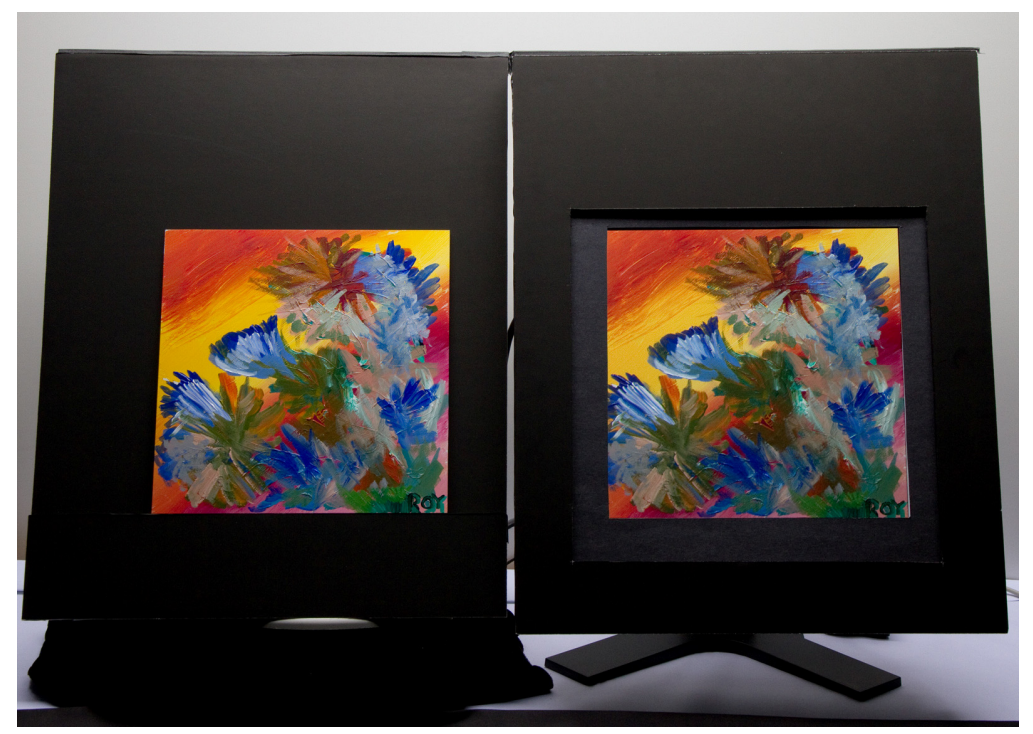

Fig. 10. Simulating surface appearance. The real painting is on the left the image produced by the system is on the right. Note the realism of the color, gloss, and texture components. As noted in the text, the image also meets the responsiveness and situatedness requirements of tangible imaging systems.

Texture: Figure 9 shows the ability of the system to simulate the shading and shadowing effects produced by surface textures. The images show renderings of the canvas and brushstroke texture of a scanned oil painting. Note that the surfaces show appropriate surface shading effects with surfaces elements oriented toward the light source appearing brighter than those facing other directions. Note also that regions that are occluded from direct illumination are appropriately shadowed. The two images show the effects of rotating the display monitor. Note that the shading and shadowing effects are different at the two orientations, correctly simulating the interactions of illumination and surface geometry. Because of the system's orientation sensor, these effects change in real-time with user manipulation of the display.

Finally, Figure 10 shows the capabilities of the system brought together in one simulation. On the left is a real oil painting with complex, spatially-varying color, gloss, and textural properties. On the right is the rendering produced by the system. Although it has been discussed earlier, it should be emphasized that rendering is interactive and dynamic, and responds as the real painting would to direct manipulation, changes in viewpoint, and changes in illumination.

\section{Applications}

The unique capabilities of tangible imaging systems could enable a wide variety of applications where natural interaction with virtual surfaces is desired. In the following 

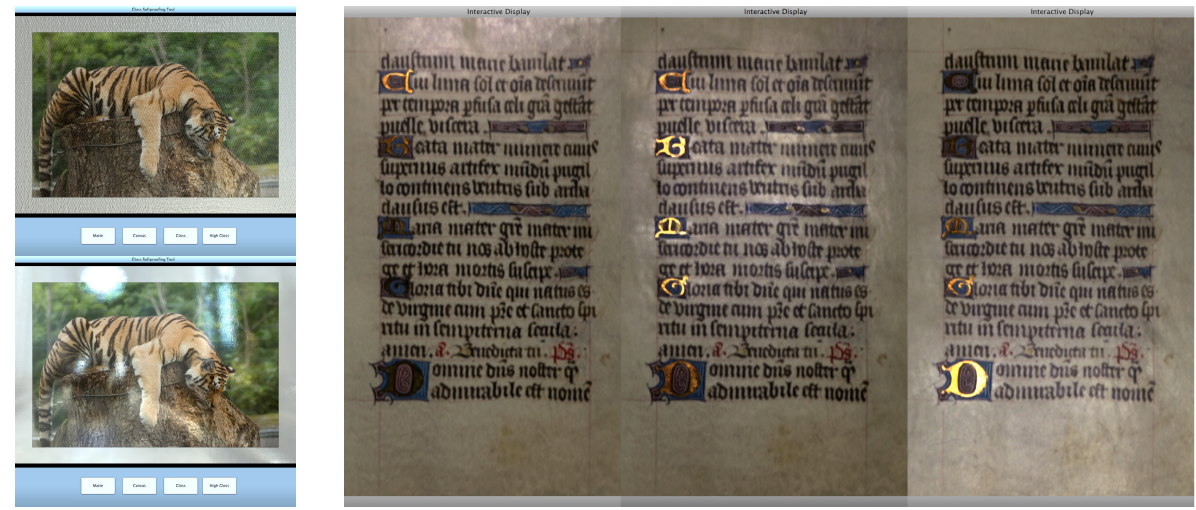

Fig. 11. Potential applications. (left) Soft-proofing of digital prints. (right) Visualization of artifacts for enhanced access to digital libraries and museums.

section we provide examples of three potential application domains: material appearance research, soft-proofing and computer-aided appearance design, and enhanced access to digital libraries and museums.

Material Appearance Research: Understanding the psychophysics of material appearance has important implications for both science and industry A major impediment to material appearance research has been the difficulty of creating stimuli that vary systematically in the properties of interest. Another limitation is the inability to dynamically control material properties, which has prevented the use of adjustment and matching procedures in experiments. Realistic computer graphics modeling and rendering methods can facilitate this, however computer graphics images on a screen typically do not support the natural modes of interaction that one uses when evaluating real materials. All of these limitations can be overcome with tangible imaging systems.

Computer-Aided Appearance Design: In printing it is valuable to be able to simulate the appearance of a hardcopy product before printing by soft-proofing on a computer display. Figure 11 shows a prototype of a soft-proofing application implemented on one of our systems [1]. With the application, users can select gloss and texture properties of papers real time, and directly manipulate the simulated print, and view it from different angles under different lighting conditions. The real-time control and natural interactivity provided by tangible imaging systems could greatly enhance the utility of the soft-proofing process. More broadly, tangible imaging systems could support computer-aided appearance design of materials like paints, coatings, and textiles.

Access to Digital Libraries and Museums: Digitization has had an enormous impact on libraries and museums. Manuscripts, paintings, and other cultural heritage objects that were once only accessible by physical visit, are now documented and accessible worldwide though digital images. However for many of these objects, static digital images are not sufficient to convey their rich and complex properties. 
Tangible imaging systems could be used to provide enhanced access to online digital collections. For example, Figure 11 shows a model of an illuminated manuscript created by Gardner et al. [26] rendered on one of our systems [1]. Using the system, a user can grasp the simulated manuscript, move it around to see the glints off the gold leaf and look at the surface from different angles to see the texture of the vellum. Tangible imaging systems provide a new interface paradigm for digital libraries and museums that could enhance access and enable advances in teaching and scholarship.

\section{Conclusion}

In this paper we have described our efforts to create tangible imaging systems that provide rich digital representations of real-world surfaces. Tangible imaging systems have three main properties: 1) the images produced by the systems are visually realistic; 2) the images are responsive to user interaction; and 3) the images are situated, appearing to be integrated with their environments. Our current system, based on a computer, LCD display, light and position sensors, and graphics rendering tools meets all these requirements; supporting the accurate simulation of the colors, glosses, and textures of surfaces with complex three-dimensional properties, and allowing users to interact with and experience these virtual surfaces as if they were real ones.

We have illustrated how tangible imaging systems can be used to advantage in a wide range of applications including material appearance research, soft-proofing and appearance design, and enhanced access to digital collections.

We are currently conducting experiments to validate the fidelity of the images produced by the system described in this paper both physically and perceptually [27]. The initial results verify the accuracy and the realism of the simulations, but the work is still in progress.

Tangible display systems represent a powerful and meaningful new approach for bridging the real and virtual worlds. The work described in this paper has described some promising first steps in this effort.

\section{References}

1. Darling, B.A., Ferwerda, J.A.: The tangiBook: a tangible display system for direct interaction with virtual surfaces. In: Proc. IS\&T 17th Color Imaging Conference, pp. 260266 (2009)

2. Darling, B.A., Ferwerda, J.A.: Tangible display systems: direct interfaces for computerbased studies of surface appearance. In: Proc. SPIE Human Vision and Electronic Imaging, vol. 7257, pp. 1-12 (2010)

3. Blatner, A.M., Ferwerda, J.A., Darling, B.A., Bailey, R.J.: TangiPaint: a tangible digital painting system. In: Proc. IS\&T 19th Color Imaging Conference, pp. 120-107 (2011)

4. http://www.tangibleimagingsystems.com

5. Sutherland, I.E.: A head-mounted three-dimensional display. Proc. AIFPS 33, 757-764 (1968) 
6. Vickers, D.L.: Sorcerer's apprentice: head-mounted display and wand. Ph.D. dissertation, Univ. of Utah (1974)

7. Callahan, M.A.: A 3-D display head-set for personalized computing. M.S. thesis, MIT (1983)

8. Fisher, S.S., McGreevy, M., Humphries, J., Robinett, W.: Virtual environment display system. In: Proc. Workshop on Interactive 3D Graphics, pp. 71-87 (1986)

9. State, A., Keller, K., Fuchs, H.: Simulation-based design and rapid prototyping of a parallax-free, orthoscopic video see-through head-mounted display. In: Proc. IEEE Computer Society of Washington, DC, pp. 28-31 (2005)

10. VPL Research, See also, http://www.vrealities.com/glove.html

11. SensAble Technologies, http://www. sensable.com

12. Ascension Technology Corp, http://www. ascension-tech.com

13. Cruz-Neira, C., Sandin, D.J., DeFanti, T.A.: Surround-screen projection-based virtual reality: the design and implementation of the CAVE. In: Proc. ACM SIGGRAPH 1993, pp. 135-142 (1993)

14. Raskar, R., Welch, G., Low, K., Bandyopadhyay, D.: Shader lamps: animating real objects with image-based illumination. In: Proc. 12th Eurographics Workshop on Rendering Techniques, pp. 89-102 (2001)

15. Raskar, R., van Baar, J., Beardsley, P., Willwacher, T., Rao, S., Forlines, C.: iLamps: geometrically aware and self-configuring projectors. In: Proc. ACM SIGGRAPH 2003, pp. 809-818 (2003)

16. Bandyopadhyay, D., Raskar, R., Fuchs, H.: Dynamic shader lamps: painting on real objects. In: Proc. International Symposium on Augmented Reality (ISAR 2001), pp. 207$216(2001)$

17. Bimber, O., Raskar, R.: Spatial augmented reality. A K Peters Ltd., Wellesley (2005)

18. Fitzmaurice, G.W.: Situated information spaces and spatially aware palmtop computers. Communications of the ACM 36(7), 39-49 (1993)

19. Tsang, M., Fitzmaurice, G.W., Kurtenbach, G., Khan, A., Buxton, W.A.S.: Boom chameleon: simultaneous capture of $3 \mathrm{D}$ viewpoint, voice and gesture annotations on a spatially-aware display. Proc. ACM UIST in CHI Letters 4(2), 111-120 (2002)

20. Francois, A.R.J., Kang, E.Y.: A handheld mirror simulation. In: Proc. International Conference on Multimedia and Expo., pp. 6-9 (2003)

21. Lazzari, M., Mclaughlin, M.L., Jaskowiak, J., Wong, W., Akbarian, M.: A haptic exhibition of daguerreotype cases for USC's Fisher Gallery. In: McLaughlin, M.L., Hespanha, J., Sukhatme, G. (eds.) Touch in Virtual Environments: Haptics and the Design of Interactive System, pp. 260-269. Prentice-Hall, Upper Saddle River (2002)

22. Ishii, H., Ullmer, B.: Tangible bits: towards seamless interfaces between people, bits and atoms. In: Proc. ACM Conference on Human Factors in Computing Systems (CHI 1997), pp. 234-241 (1997)

23. Buxton, W.: Surface and tangible computing, and the small matter of people and design. In: IEEE International Solid-State Circuits Conference Digest of Technical Papers, vol. 51, pp. 24-29 (2008)

24. Darling, B.A., Ferwerda, J.A.: Real-time multispectral rendering with complex illumination. In: Proc. IS\&T 19th Color Imaging Conference, pp. 345-351 (2011)

25. Ward, G.J.: Measuring and modeling anistropic reflection. Proc. ACM SIGGRAPH 1992, Computer Graphics 26(2), 265-272 (1992)

26. Gardner, A., Tchou, C., Hawkins, T., Debevec, P.: Linear light source reflectometry. Proc. SIGGRAPH 2003, ACM Transactions on Graphics 22(3), 749-758 (2003)

27. Darling, B.A., Ferwerda, J.: Seeing virtual objects: simulating reflective surfaces on emissive displays. In: Proc. IS\&T 20th Color Imaging Conference, pp. 1-7 (2012) 